\title{
Electrical Properties of Double Perovskite LaPbFeTiO6 Synthesized by Sol-Gel Method
}

K. Iben Nassar ( $\square$ kaisibnnassar12@gmail.com )

Université de Sfax

M. Slimi

Université de Sfax

N. Rammeh

Université de Sfax

Manuel. P. F. Graça

University of Aveiro

\section{Research Article}

Keywords: Double perovskite, Sol-gel method, X-ray diffraction, Complex impedance, dielectric relaxation, conductivity

Posted Date: July 27th, 2021

DOI: https://doi.org/10.21203/rs.3.rs-740214/v1

License: (c) (1) This work is licensed under a Creative Commons Attribution 4.0 International License.

Read Full License 


\title{
Electrical properties of double perovskite $\mathrm{LaPbFeTiO}_{6}$
}

\section{synthesized by sol-gel method}

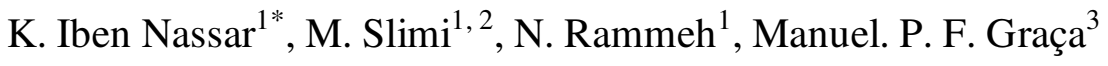 \\ ${ }^{1}$ Laboratoire de physique des matériaux, Faculté des Sciences de Sfax, B.P. 1171, 3000 Sfax, \\ Université de Sfax, Tunisie \\ ${ }^{2}$ Faculté des Sciences de Monastir, Université de Monastir 5019 Monastir, Tunisie. \\ ${ }^{3}$ I3N-Aveiro, Department of Physics, University of Aveiro, 3810-193, Aveiro, Portugal
}

\begin{abstract}
The double perovskite structure is interesting for many researches in various fields of physics and their technological applications. The $\mathrm{LaPbFeTiO}_{6}$ (LPFTO) compound is a very important for many technologies because it has dielectric and electrical properties. It has made an attractive semiconductor material for the electronic field, such as the realization of filters or capacitors. In this work, $\mathrm{LaPbFeTiO}_{6}$ was prepared by the sol-gel method.The structural, morphological and electrical properties of this sample were studied using different techniques. $X$-ray diffraction analysis, the sample crystallized in the rhombohedral system with space group $\mathrm{R} \overline{3} \mathrm{C}$. The surface of the sample and the average grain size were examined by scanning electron microscope (SEM). The electrical conductivity and dielectric characterizations were studied as a function of frequency and temperature (200-380K) by impedance spectroscopy. Indeed, the dielectric constant increases with increasing temperature. The AC conductivity obeys the Jonscher power law. The activation energy obtained from the complex impedance and the conductivity are close. The frequency dependence of the electrical impedance shows the existence of a relaxation phenomenon.
\end{abstract}

Keywords: Double perovskite; Sol-gel method; X-ray diffraction; Complex impedance; dielectric relaxation; conductivity

* Correspondent author: Kaisibnnassar12@gmail.com

\section{Introduction}

Double perovskite compounds have the general formula $\mathrm{A}_{2} \mathrm{~B}^{\prime} \mathrm{B}^{\prime \prime} \mathrm{O}_{6}$ or $\mathrm{A}^{\prime} \mathrm{A}^{\prime \prime} \mathrm{B}^{\prime} \mathrm{B}^{\prime \prime} \mathrm{O}_{6}$, where the cations occupying the A site of the perovskite are essentially alkaline earth or rare earth metal ions, while the $\mathrm{B}^{\prime}$ and $\mathrm{B}^{\prime \prime}$ sites are two transition metals that can be ordered to 
octahedral sites thus forming the octahedra $\mathrm{B}^{\prime} \mathrm{O}_{6}$ and $\mathrm{B}^{\prime \prime} \mathrm{O}_{6}$. These materials have attracted a lot of interest in recent years because of their variety of physical properties. Varying the choice of $\mathrm{B}^{\prime}$ and $\mathrm{B} "$ cations, these compounds present a wide variety of electrical and magnetic properties, namely, metallicity, semi-metallicity, insulation as well as ferromagnetism (FM), ferrimagnetism (FIM) and antiferromagnetism (AFM) [1,2]. Multiferroic materials exhibit more than one ferroic order. In particular, materials in which ferromagnetic and ferroelectric orders coexist are attracting much attention in fundamental physics and chemistry [3-5]. In the case of ferroelectric, the word ferro indicates that a material has a permanent electric polarization even in the absence of an electric field and a residual magnetization that can be reversed by the application of the magnetic field in the case of ferromagnetic. Moreover, the first studies are devoted to oxides of types $\operatorname{Ln}_{1-\mathrm{x}} \mathrm{A}_{\mathrm{x}} \mathrm{MnO}_{3}, \mathrm{x} \in$ $[0,1](\mathrm{Ln}=$ terre rare, $\mathrm{A}=\mathrm{Ca}, \mathrm{Ba}, \mathrm{Sr} . .).[6,7]$. Manganese has very important magnetic properties (ferromagnetic and antiferromagnetic).

Indeed, important theoretical advances, with the development of new concepts in solid state physics, have been actively studied on these oxides. These studies have been the basis for the development of the double exchange (DE) [8] and super exchange (SE) [9] theories. Indeed, the first material used for dielectric properties was Rochelle salt, used in phonographs, currently lead-based perovskites, such as $\mathrm{Pb}\left(\mathrm{Zr}_{\mathrm{x}} \mathrm{Ti}_{1-\mathrm{x}}\right) \mathrm{O}_{3}(\mathrm{PZT})$ or $\mathrm{Pb}\left(\mathrm{Mg}_{1 / 3} \mathrm{Nb}_{2 / 3}\right) \mathrm{O}_{3}-\mathrm{PbTiO}$ (PMN-PT) [10-14], are the most used. These materials have the best piezoelectric and ferroelectric properties with limited fabrication cost.

Moreover, researchers have become interested in double perovskite compounds due to their different physical properties, which can be adjusted by applying electric and magnetic fields; ferroelectric perovskites are examples of such interesting compounds; they are used in various technological applications such as non-volatile memories, sensors, capacitors, high voltage line insulation, electric motor spark plugs, circuit breakers and switches, microwave dielectric filters and pyroelectric detectors. Thus, perovskites have a wide application in modern electronics due to their high dielectric permittivity, high piezoelectric coefficient, ferroelectricity, semiconductivity, catalytic activity and thermoelectricity.

One of the main objectives of this work was to prepare a new double perovskite LPFTO material by the sol-gel route and to study the structural, morphological and electrical properties. The dependence of the electrical conductivity and dielectric constant on temperature and frequency was studied for the sample in the range of the setting. This was 
done keeping in mind that it is a very interesting material with high permittivity to be used in electronics and energy storage.

\section{Experimental Details}

\subsection{Sample Preparation}

The sample LPFTO was prepared by sol - gel as shown in Fig. 1. The various precursors were dissolved in nitric acid and water distilled at $120^{\circ} \mathrm{C}$ for $4 \mathrm{~h}$ with stirring magnetic mixer. Then, citric acid and ethylene glycol are added. The obtained solution was dried and calcined to obtain a nano-crystalline powder. The material was crushed and pressed into a pellet and then sintered. Finally, it was annealed between $600{ }^{\circ} \mathrm{C}$ and $1000{ }^{\circ} \mathrm{C}$ for $10 \mathrm{~h}$ to obtain a homogeneous material.

\subsection{Sample Characterization}

However, the powder was characterized by X-ray diffraction (XRD), using an X'Pert MPD Philips diffractometer ( $\mathrm{CuK} \alpha$ radiation, $\lambda=1.54060 \AA$ ) was performed at room temperature. The surface morphology of the sample was analyzed by scanning electron microscopy (SEM), using a TESCAN Vega 3 microscope. Before microscopic observation, the sample was coated with carbon to promote surface conductivity.

For electrical measurements, the sample was prepared as a disk, which was configured as a planar capacitor, where the opposing surfaces were painted with a conductive silver paste that forms two electrodes. The impedance spectroscopy was performed using a precision impedance analyzer Agilent 4294A, in Cp-Rp configuration (capacitance in parallel with resistance), in the frequency range of $100 \mathrm{~Hz}-1 \mathrm{MHz}$ and temperature from 200 to $380 \mathrm{~K}$, using a cryostat-bath in which the sample is in a helium atmosphere to improve heat transfer and avoid humidity.

\section{Results and Discussion}

\subsection{XRD Analysis and Morphological Characterisation}

Fig. 2 shows the X-ray diffraction (XRD) pattern of $\mathrm{LaPbFeTiO}_{6}$. From the figure, there is no secondary phases detected, which shows that the material is a single-phase and well crystallized. It crystallizes in the rhombohedral system with the space group $\mathrm{R} \overline{3} \mathrm{C}$ and there is an accordance between the calculated and measured intensities which shows the good crystallization. The refined structural parameters for the sample were found to $b e \mathrm{a}=\mathrm{b}=5.5655$ 
$\AA, \mathrm{c}=13.5861 \AA, \mathrm{V}=364.4466 \AA^{3}$ and the reliability factors were found $\chi^{2}=1.58, \mathrm{R}_{\mathrm{f}}=1.46$ and Bragg factor $R_{B}=1.96$. In the context of the morphological study of grains, the mean crystallite size calculated using Debye - Scherrer formula [15]:

$$
D=\frac{0.9 \cdot \lambda}{\beta \cdot \cos \theta}
$$

where, $\mathrm{D}$ is average size of crystallites, $\lambda$ is wavelength (1.54060 $\AA$ ), $\beta$ is width at mid-height of the peak and $\theta$ is the diffraction angle of the peak considered. We get the grain size is 370 nm. Fig. 3 shows the LPFTO surface morphology recorded for a gold-plated bulk pellet sample using a scanning electron microscope (SEM). The nature and size of the microstructures of the sample suggest that the surface is very dense due to the uniform grain distribution separated by grain boundaries with different size ranges. The grain size is equal to $366 \mathrm{~nm}$, which is an agreement with the particle size, calculated from the XRD results.

\subsection{Impedance Spectroscopy and Dielectric Study}

Fig. 4 shows the temperature-dependence of the complex impedance spectra (Nyquist plot) ( $Z^{\prime \prime}$ vs. $\left.Z^{\prime}\right)$ of the prepared sample. The spectra in this graph are characterized by semicircle arcs centered on the real axis $Z^{\prime}$. These arcs represent the grain and the grain boundary, respectively. We deduce a non-Debye type relaxation [16, 17]. Indeed, the increase of the temperature indicates the decrease of the diameter of the semicircular arcs, which explains a semiconducting behavior of this material [17]. The impedance decreases with the increase of temperature, causing the displacement of charge carriers involved in the conduction mechanism [16]. The different spectra are refined using the Zview software. Therefore, the experimental data of the LPFTO compound are modeled by an equivalent circuit composed of three elements $(\mathrm{R}, \mathrm{C}$ and $\mathrm{CPE})$ in parallel. The constant phase element $\left(\mathrm{Z}_{\mathrm{CPE}}\right)$ is determined from the following equation [18]:

$$
Z_{C P E}=\frac{1}{Q(j w)^{\alpha}}
$$

Or, CPE is the fractal capacitance was used instead of a pure capacitance to account for the decentering of the associated semicircle from the $\mathrm{Z}^{\prime}$ axis [19]. More generally, the use of a FPC reflects deviations from an ideal dielectric behavior and thus from a time distribution. Therefore, the fractal capacitance is characterized by two parameters: a coefficient $Q$ and an exponent $\alpha(0 \leq \alpha \leq 1)$, which represents the amount of deviation from an ideal system. The 
parameters of the equivalent electric circuit corresponding to the compound under study is deduced from a good quality of the "fit" we performed and are summarized in Table 1.

The frequency dependence of the real part of impedance $\left(Z^{\prime}\right)$ at different temperatures is shown in Fig. 5. It is observed that $Z^{\prime}$ for low frequencies, is larger at low temperature than at high temperature, indicating that the conductivity increases with temperature. But, for high frequencies from $10^{5} \mathrm{~Hz}$, the values of the latter gradually decrease and merge, probably due to the reduction of the barrier at high temperature, which was compensated by the release of space charges $[16,17]$.

Fig. 6 shows variation of the imaginary part of the impedance $\left(Z^{\prime \prime}\right)$ at different temperatures. It is clear that $\left(Z^{\prime \prime}\right)$ decreases with increasing frequency and increasing temperature. $Z$ " reaches a maximum at a specific frequency $\left(Z_{\max }\right)$, due to the relaxation of the material [16]. This maximum frequency is called the relaxation frequency $\mathrm{f}_{\max }$. The inverse of this is known as the relaxation time $(\tau)$. According to the figure, the relaxation peaks drift towards the high frequency region, which indicates a decrease of the relaxation time with increasing temperature. In addition, this relaxation phenomenon is probably due to defects at higher temperatures and immobile species at lower temperatures [19]. The relaxation time is given by the following Arrhenius law [16-19]:

$$
\tau=\tau_{0} \exp \left(\frac{-E_{a}}{K_{B} T}\right)
$$

Where Ea is the activation energy, $\tau_{0}$ is the characteristic relaxation time, and $K_{B}$ is the Boltzmann constant. The activation energy is defined by the energy required for the charge carrier to jump between neighboring sites [20]. We obtain a value equal to $0.32 \mathrm{eV}$ and $\tau_{0}=$ $2.3610^{-10} \mathrm{~s}$. To explain the dielectric relaxation of the material, we plot the normalized spectrum of the imaginary $Z^{\prime \prime} / Z_{\max }$ part of the impedance in Fig. 7. All the normalized spectra do not overlap, indicating that the dielectric relaxation is temperature dependent [21]. It is observed that all the spectra shift to higher frequencies as the temperature increases.

The frequency dependence of the real part of permittivity dielectric is shown in Fig. 8. The values of $\varepsilon^{\prime}$ increase globally with temperature; this increase is much more marked for frequencies below $10^{3} \mathrm{~Hz}$. This is due to the accumulation of charges at the interfaces between the sample and the electrodes. On the other hand, the real part is relatively high at low frequency and decreases with increasing frequency, indicating dielectric dispersion. Furthermore, the dielectric behavior is explained by a polarization process belonging to the 
grain boundary contribution. At low frequencies, charge carriers are accumulated in the grain boundary and the hopping requires more energy, which increases the dielectric constant [22]. The highest value of $\varepsilon^{\prime}$ obtained at room temperature is useful for capacitor, transducer and microwave applications. Therefore, this material is important in the electronic field because of its high permittivity.

\subsection{Conductivity Study}

In order to analyze the dynamic response of the material as a function of the applied electric field, the study of the experimental data was performed on the conductivity, also called alternating current conductivity $\left(\sigma_{\mathrm{AC}}\right)$, this conductivity is associated with the conduction of electric charges whose direction is in phase with the applied electric field and their frequency dependence also provides information on the nature of the charge carriers. The frequency dependence of the conductivity follows the Jonscher'spower law [23]:

$$
\sigma(\omega)=\sigma_{D C}+A \omega^{s}
$$

where $\omega$ is the angular frequency, $\sigma_{\mathrm{DC}}$ is the conductivity, $\mathrm{A}$ is a constant and s represents the interaction between the mobile ions and the surrounding lattices, which can have a value between zero and unity. Fig.9 shows the variation of $\sigma_{\mathrm{AC}}$ of the sample with frequency at different temperatures. The conductivity curves can be divided into two parts. The first part (the low frequency region), represents the behavior where the conductivity curves show a frequency independent plateau which has been assigned to the DC conductivity. The second part (the high frequency region), represents the AC conductivity, which increases progressively with frequency. Indeed, the conductivity increases with temperature, which may be caused by an intensification of the charge carrier hopping process [22, 23]. According to Jonscher [24], the variation in high-frequency conductivity is at the origin of the relaxation phenomenon in the material which is due to the mobility of the charge carriers, that is to say the displacement towards a new site at from the original position. We performed the fit in Fig. 9 using equation (4) with ORIGIN 8.0 software; the experimental results are summarized in Table 2. This result can be explained that the material is semi-conductive. Fig.10, illustrates the dependence of DC conductivity and linear $\operatorname{Ln}\left(\sigma_{D C}\right)$ as a function of $1000 / T$, the activation energy can be obtained from the Arrhenius equation follows the Arrhenius law [23, 25]:

$$
\sigma(T)=\sigma_{0} \exp \left(\frac{-E_{a}}{K_{B} T}\right)
$$


where $\mathrm{Ea}$ is the activation energies, $\sigma_{0}$ is the pre-exponential term and $\mathrm{K}_{\mathrm{B}}$ is the Boltzmann's constant. Starting from equation 5 , it is then possible to write:

$$
\operatorname{Ln}[\sigma(T)]=-\frac{E_{a}}{K_{B} T}+\operatorname{Ln}\left(\sigma_{0}\right)(6)
$$

The activation energy can then be determined by calculating the slope of the line representing: $\operatorname{Ln}[\sigma(T)]=f\left(\frac{1}{T}\right)$. The activation energy value obtained for LaPbFeTiO6 is $0.29 \mathrm{eV}$, it is clear that the activation energy values obtained from the conductivity and dielectric relaxation analysis are very close, indicating that the electric charge carriers originate from the hopping mechanism [26]. In the region of higher frequencies and temperatures, the conductivity values are large, which is attributable to a higher mobility of the charge carriers. The mobility of $\mu$ carriers is defined by the following relationship [27]:

$$
\mu=\frac{\sigma_{D C} M}{N_{A} \rho n e}
$$

where $\rho$ is the density, $M$ is the molecular weight of the sample, $n$ is the number of charge carriers, and e is the elementary charge. The temperature dependence of the charge carrier mobility of the sample is shown in Fig.11. It can be seen that the charge carrier mobility increases with temperature. The motion is less than that of electrons and holes [20], except for the electron motion and/or the hole motion of the DC conduction mechanism. Finally, from the AC conductivity mechanism, we can deduce the variation of the exponent s [22]. The aim of our study is to identify the conduction model that allows us to understand the electric charge transport phenomena in the studied material. We consider the temperature dependence of this parameter in the same Fig.11. S decreases with increasing temperature, indicating a dominant conduction mechanism [28]. When $\mathrm{s}<1$, it indicates a transitive oscillation motion, while s> 1 suggests a localized jump [24]. From Figure 11, the material exhibits a localized hopping mechanism throughout the studied temperature range. However, this parameter decreases with increasing temperature, suggesting that the Correlated Barrier Hopping or CBH model [29, 30]. The model is introduced by Pike [31] and then modified by Elliott [32], which describes the hopping of a charge carrier over an energy barrier. A favorable hopping between two states no longer depends only on the distance or the energy difference, but on a correlation between the two values. 


\section{Conclusion}

In this work, we successfully synthesized the ceramic material $\mathrm{LaPbFeTiO}_{6}$ by sol-gel method. XRD and SEM studies at room temperature confirmed that the material is singlephase, crystallizes in the rhombohedral system with the space group $\mathrm{R} \overline{\mathrm{C}} \mathrm{C}$ and very dense. The impedance spectroscopy analysis was used at a temperature of 200-380 K and at a frequency up to $1 \mathrm{MHz}$; it shows the appearance of a relaxation phenomenon of the material. The dielectric study confirms that the sample has a high permittivity; the values of the dielectric constant suggest that the material can be used for the electronic field in technological applications. The AC conductivity obeys the Jonscher law. The activation energy values are estimated from the analysis of the electrical conductivity and relaxation are very close, indicating a common electrical transport mechanism. Finally, activation energy calculations revealed a semiconducting behavior of LPFTO. The variation of the exponent $s$ with temperature confirms the correlated barrier hopping conduction model $\mathrm{CBH}$. 


\section{References:}

[1] Anderson, M.T, Greenwood, K.B, Taylor, G.A, Poeppelmeier, K.R. Prog. Solid State Chem. 22, 197-233 (1993).

[2] Philip, J.B. et al, Phys. Rev. B 68, 144431 (2003).

[3] Ramesh, R.; Spaldin, N.A. Nat. Mater. 2007, 6, 21-29.

[4] Fiebig, M.; Spaldin, N.A. Eur. Phys. J. B. 2009, 71, 293-297.

[5] Spaldin, N.A.; Cheong, S.-W.; Ramesh, R. Phys. Today 2010, 63, 38-43.

[6] M. B. Salomon et M. Jaime, Rev. Mod. Phys. 73, 583 (2001);

[7] E. L. Nagaev, Phys. Rep. 346, 387 (2001).

[8] S. Jin, T.H. Tiefel, M. McCormack, R. Ramesh et L.H. Chen, Science. 264, 413 (1994).

[9] C. Zener, Phys. Rev. 82 (1951) 403.

[10] X.L. Chao, D.F. Ma, R. Gu, Z.P. Yang, J. Alloys Compd. 491 (2010) 698-702.

[11] X.L. Chao, Z.P. Yang, Y.F. Chang, M.Y. Dong, J. Alloys Compd. 477 (2009) 243-249.

[12] H. Hao, S.J. Zhang, H.X. Liu, T.R. Shrout, J. Appl. Phys. 105 (2009) 024104.

[13] Z.G. Zhu, B.S. Li, G.R. Li, W.Z. Zhang, Q.R. Yin, Mater. Sci. Eng. B 117 (2005) 216220.

[14] Q. Li, Y. Yang, D.D. Wan, Y. Cheng, M. Zha, Mater. Sci. Eng. B 163 (2009) 145-150

[15] M. A. Ahmed, A. A. Azab and E. H. El-Khawas, J. Mater. Sci.: Mater. Electron., 2015, $26,8765-8773$.

[16] A. Omri, M. Bejar, M. Es-Souni, M. A. Valente, M. P. F. Graça and L. C. Costa, J. Alloys Compd., 2012, 536, 173-178.

[17] M. M. Costa, G. F. M. Pires J'unior and A. S. B. Sombra, Mater. Chem. Phys., 2010, $123,35-39$.

[18] Y. Olofsson, J. Groot, T. Katrašnik, e G. Tavcar, in Electric Vehicle Conference (IEVC), 2014 IEEE International, pp. 1-6, 2014.

[19] K. Lily, K. P. Kumari, K. Prasad and R. N. P. Choudhary, J. Alloys Compd., 2008, 453, $325-331$.

[20] Z. Mohamed, R. Brahem, J. Dhahri, K. Khirouni and E. K. Hlil, Phase Transitions, 2016, 89, 958-969.

[21] R. Gerhardt, J. Phys. Chem. Solids, 1994, 55, 1491- 1506.

[22] R. Adrian, L. Dariusz, K. Marek, M. Jaroslaw and W. Patryk, Materials, 2018, 11, 735.

[23] R. Kumari, N. Ahlawat, A. Agarwal, S. Sanghi and M. Sindhu, J. Alloys Compd., 2017, $695,3282-3289$. 
[24] A.K. Jonscher, Nature 267, 673 (1977).

[25] B. C. Sutar, R. N. P. Choudhary and P. R. Das, Ceram. Int., 2014, 40, 7791-7798.

[26] A. Rahal, S. M. Borchani, K. Guidara and M. Megdiche, R. Soc. Open Sci., 2018, 5, 171472, DOI: 10.1098/rsos.171472.

[27] R. K. Panda, R. Muduli, S. K. Kar and D. Behera, J. Alloys Compd., 2014, 615, 899905.

[28] V. L. Mathe, K. K. Patankar, S. D. Lotke, P. B. Joshi and S. A. Patil, Bull. Mater. Sci., $2002,25,347-350$.

[29] K. Funke; Prog. Solid State Chem. 22 (1993) 111.

[30] K. Shimakawa, Philosophical Magazine B 46 (1982) 123.

Rockstad, H. K. \& Pike, G. E. Comment on 'ac conductivity of scandium oxide and a new hopping model for conductivity'. Phys. Rev. B 8, 4026-4027 (1973).

[31] Rockstad, H. K. \& Pike, G. E. Comment on 'ac conductivity of scandium oxide and a new hopping model for conductivity'. Phys. Rev. B 8, 4026-4027 (1973).

[32] Elliott, S. R. A theory of a.c. conduction in chalcogenide glasses. Philos. Mag. 36, 12911304 (1977). 


\section{Figures Captions}

Fig. 1 Schematic illustration of the synthesis of LaPbFeTiO6 powder by sol-gel method.

Fig. 2: X-ray powder diffraction of LaPbFeTiO6 recorded with the $\mathrm{Cu}$ Ka radiation.

Fig. 3: SEM images of $\mathrm{LaPbFeTiO}_{6}$.

Fig. 4: Nyquist plots of the $\mathrm{LaPbFeTiO}_{6}$.

Fig. 5: Variation of real part of the impedance as a function of frequency at various temperatures.

Fig. 6: Variation of imaginary part of the impedance as a function of frequency at various temperatures.

Fig.7: The normalized spectra of the imaginary impedance $Z^{\prime \prime} / Z_{\max }$ at different temperatures.

Fig. 8: Frequency and temperature dependencies of the dielectric constant of $\mathrm{LaPbFeTiO}_{6}$.

Fig.9: Frequency and temperature dependencies of the electrical conductivity of the $\mathrm{LaPbFeTiO}_{6}$.

Fig.10: Temperature dependence of the DC conductivity of the $\mathrm{LaPbFeTiO}_{6}$ ceramic and the linear $\ln \left(\sigma_{\mathrm{DC}}\right)$ as a function of $1000 / \mathrm{T}$, which satisfy the Arrhenius law.

Fig.11: Temperature dependences of the charge carriers mobilities and the exponent $s$ of the $\mathrm{LaPbFeTiO}_{6}$. 


\section{Table captions}

Table 1 : Parameters of refinements of the equivalent circuit proposed for the compound $\mathrm{LaPbFeTiO}_{6}$.

Table 2 : Results of adjustment of Jonscher power-law of the alternative conductivity for the compound $\mathrm{LaPbFeTiO}_{6}$. 


\section{Figures}

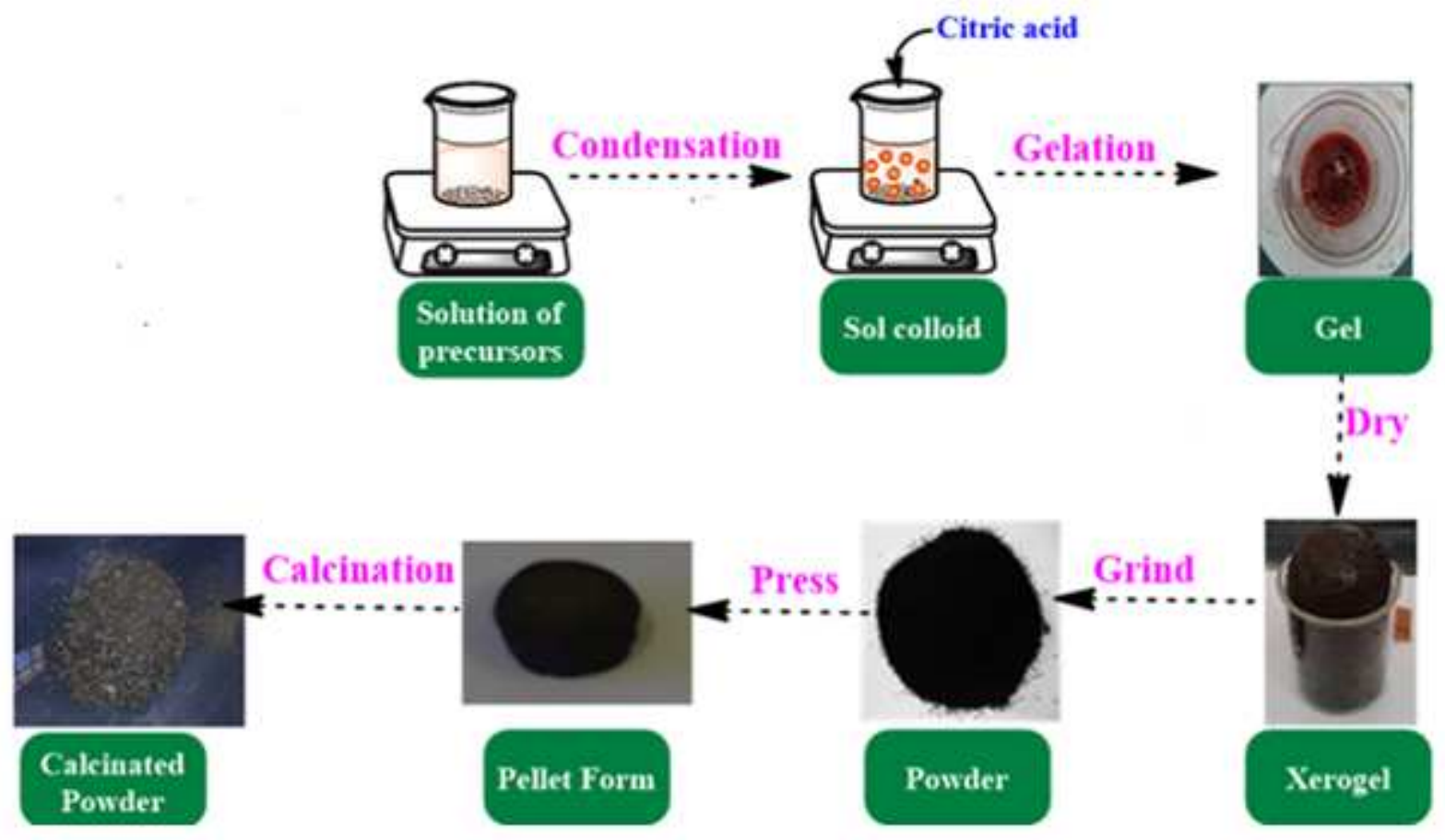

Fig.1

\section{Figure 1}

Schematic illustration of the synthesis of LaPbFeTiO6 powder by sol-gel method. 


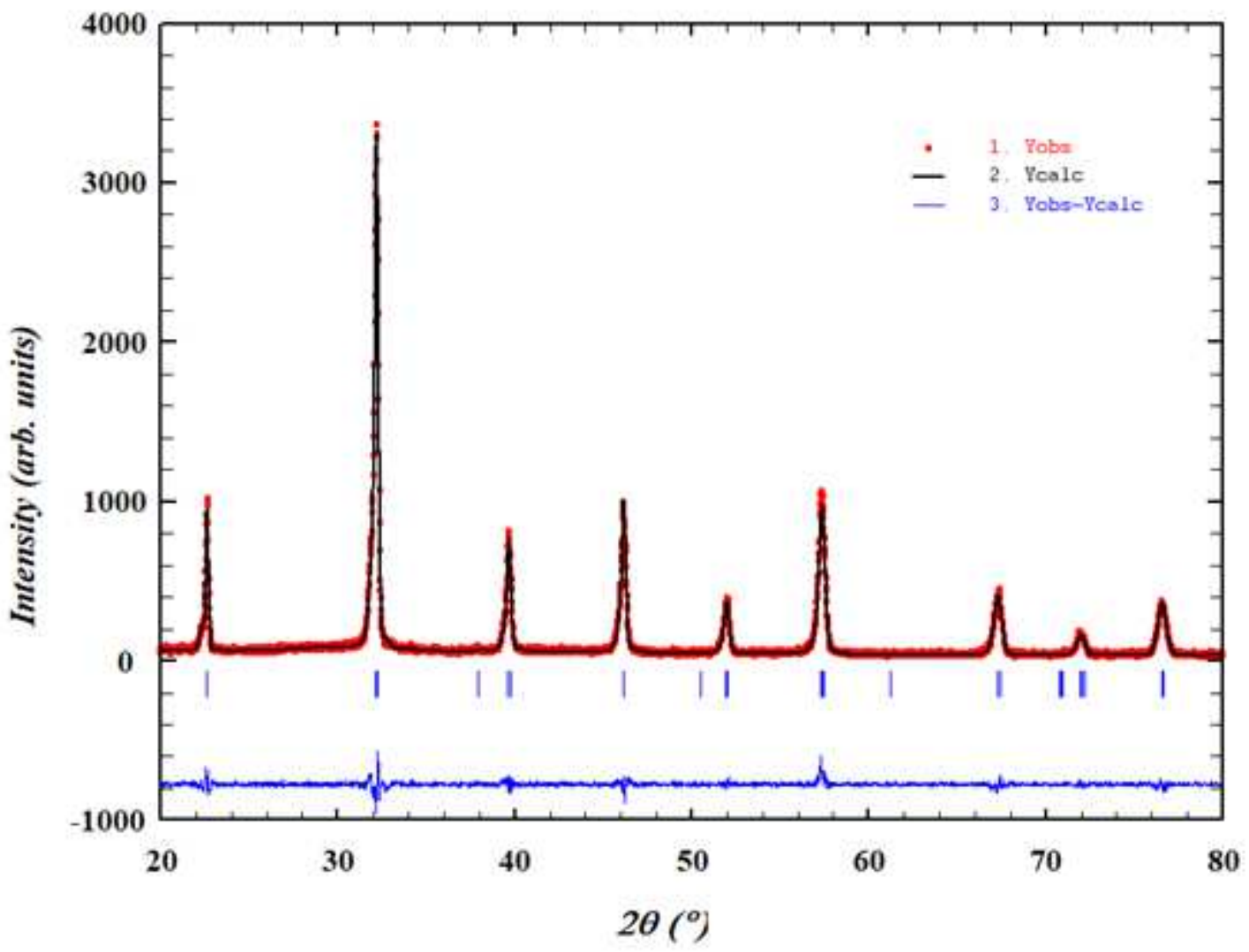

Fig.2

Figure 2

X-ray powder diffraction of $\mathrm{LaPbFeTiO6}$ recorded with the $\mathrm{Cu}$ Ka radiation.
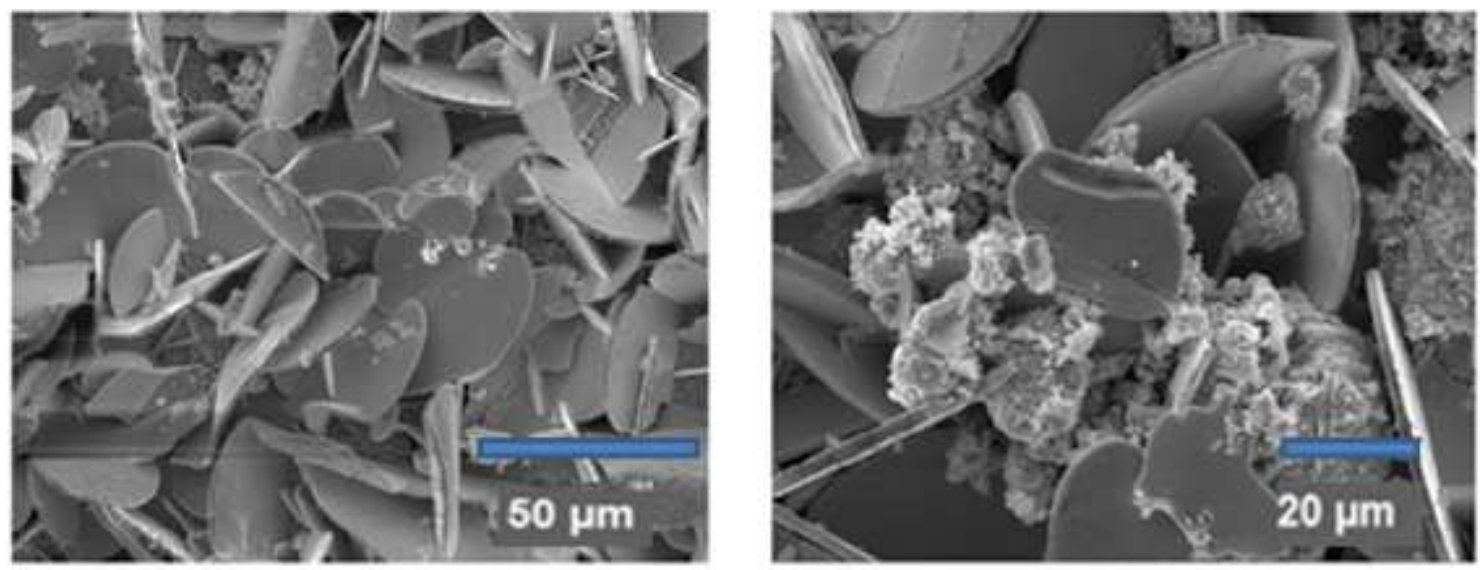

Fig.3

Figure 3 
SEM images of LaPbFeTiO6.

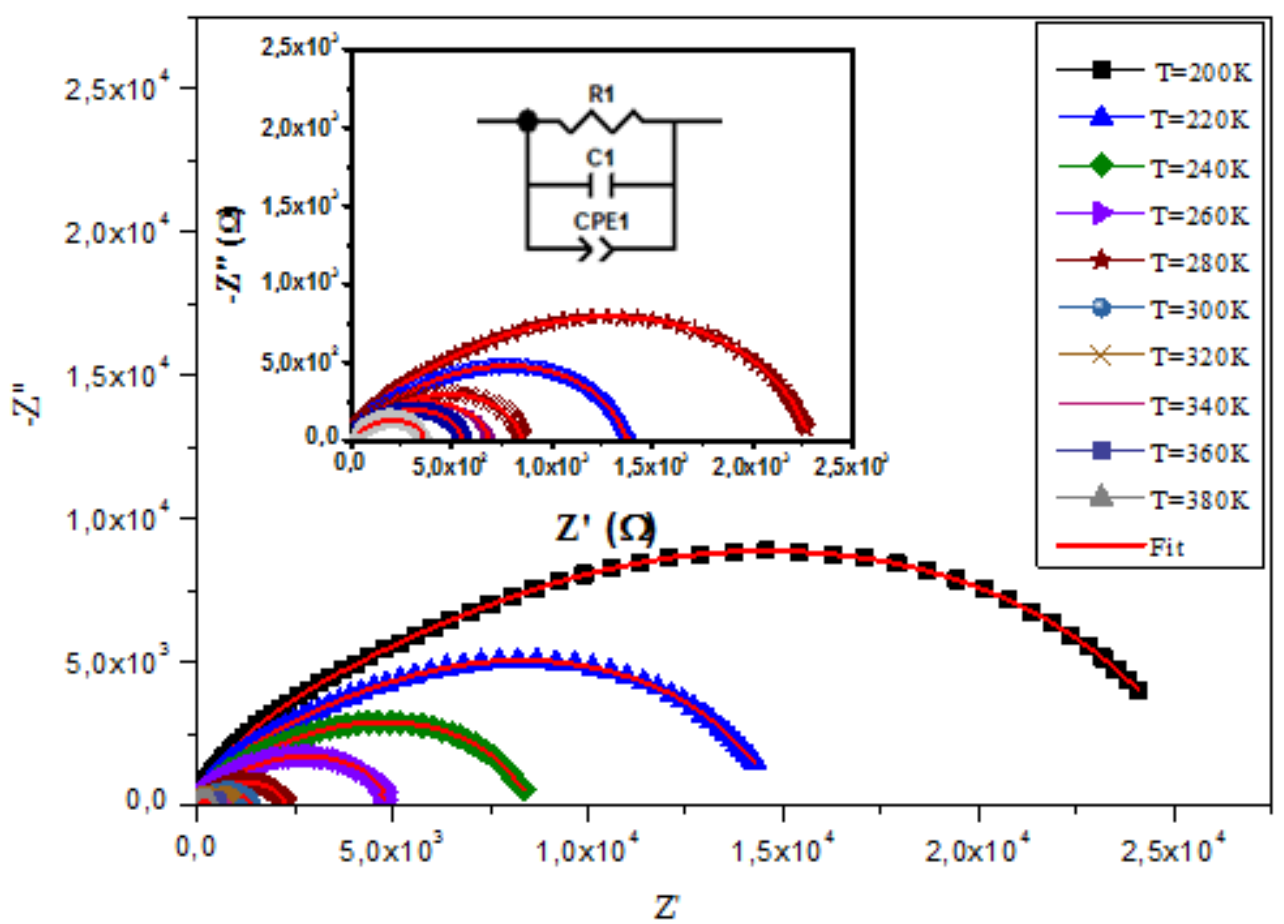

Fig.4

Figure 4

Nyquist plots of the LaPbFeTiO6. 


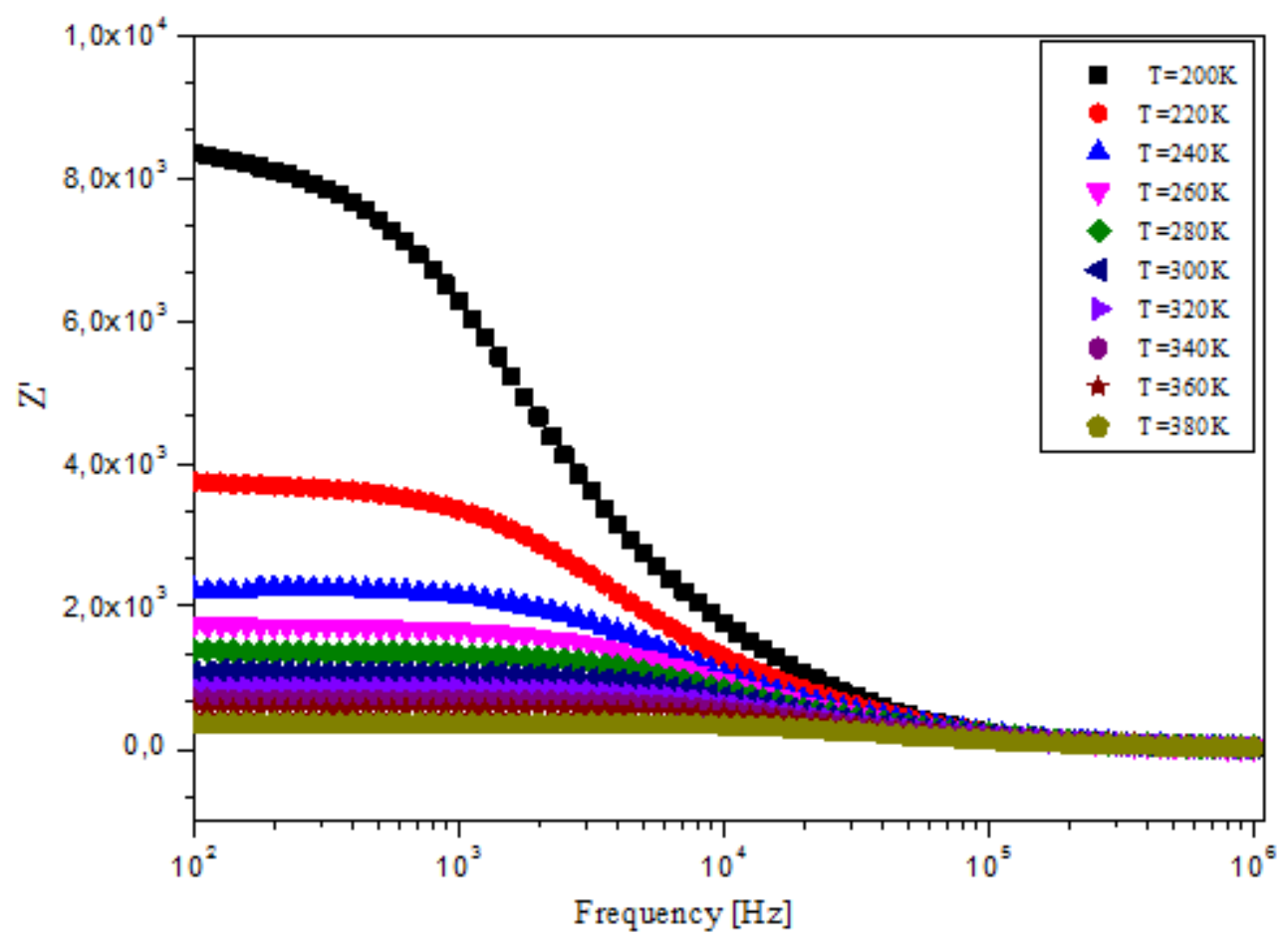

Fig.5

Figure 5

Variation of real part of the impedance as a function of frequency at various temperatures. 


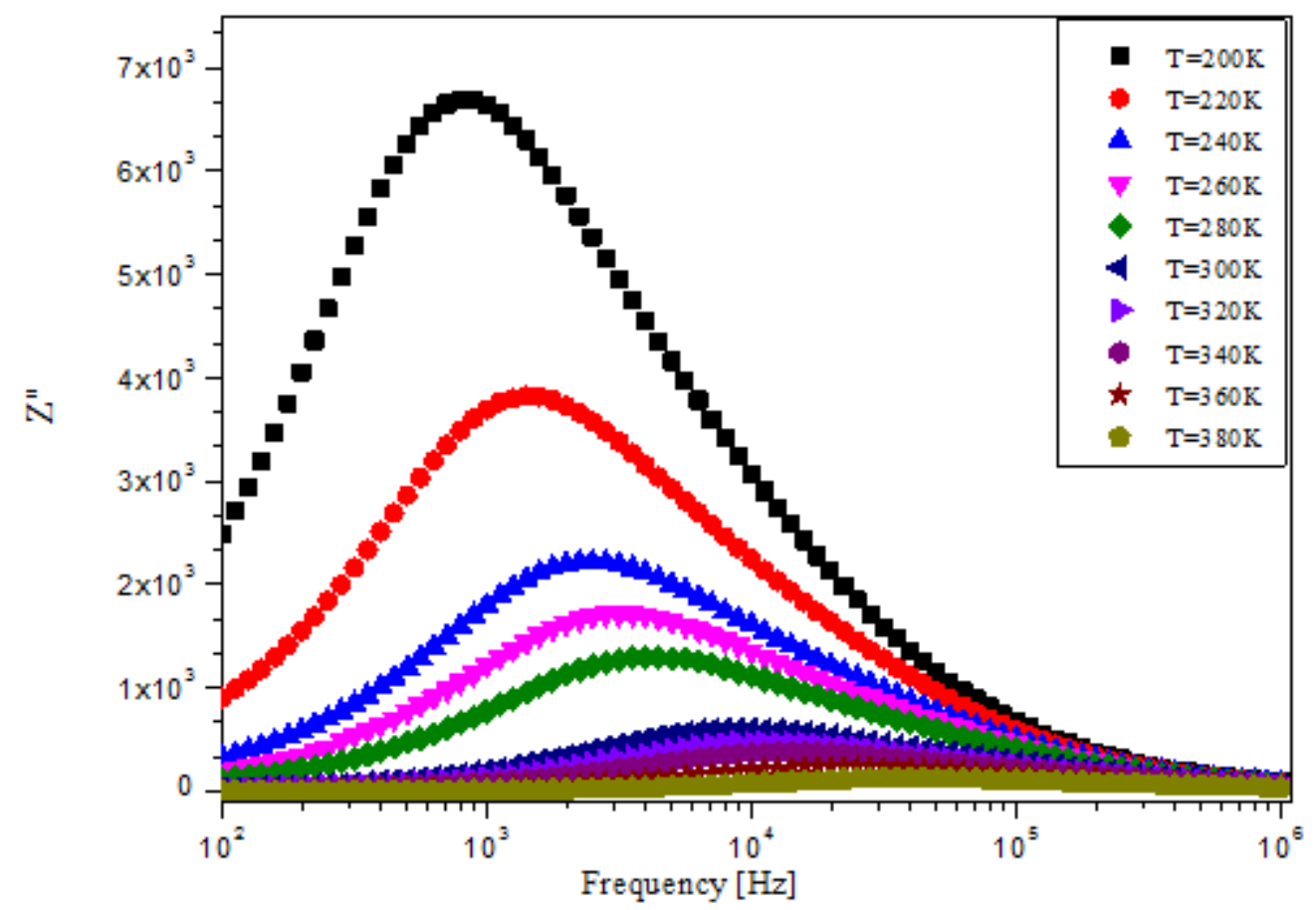

Figure 6

Variation of imaginary part of the impedance as a function of frequency at various temperatures. 


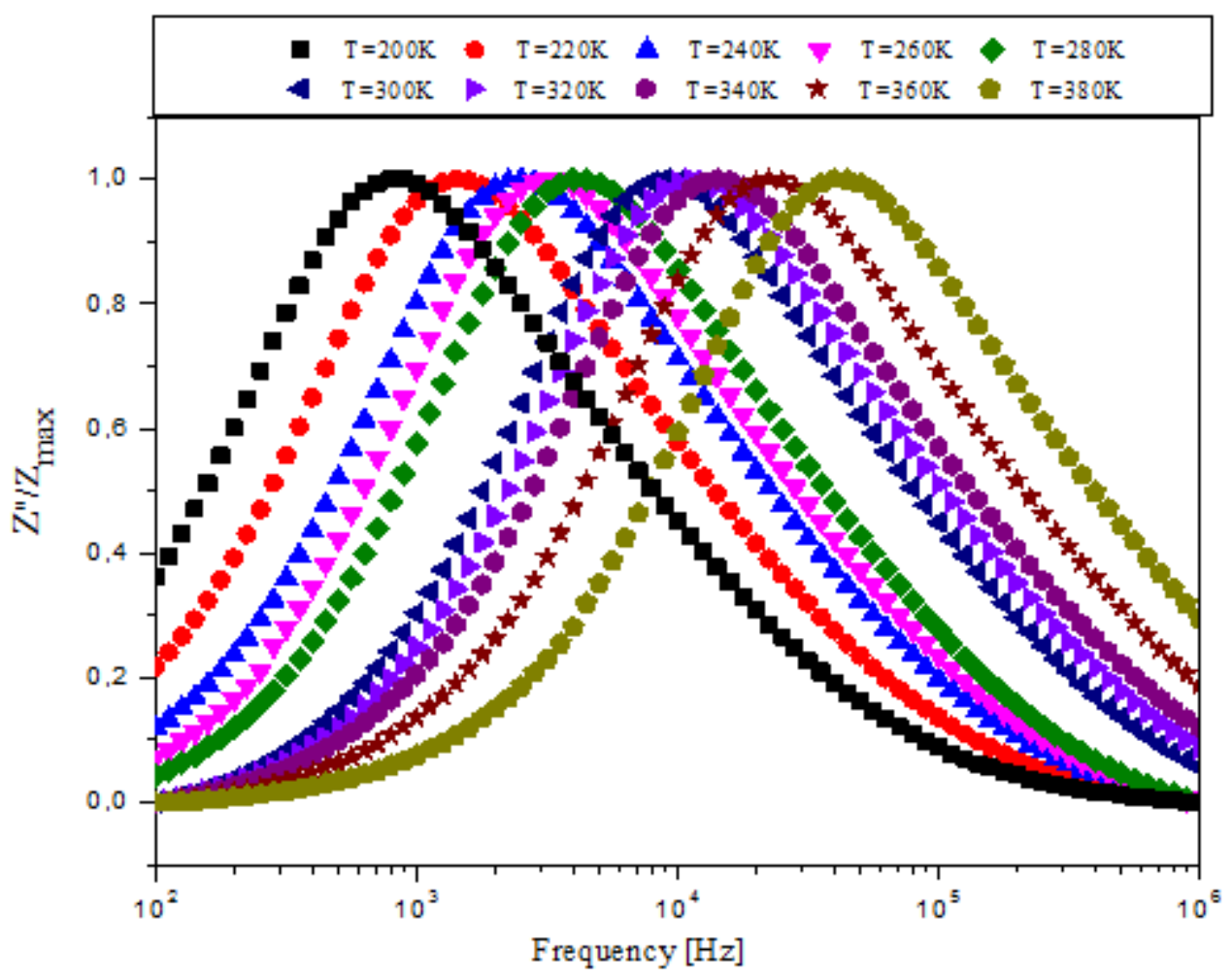

Fig.7

Figure 7

The normalized spectra of the imaginary impedance Z"/Zmax at different temperatures.

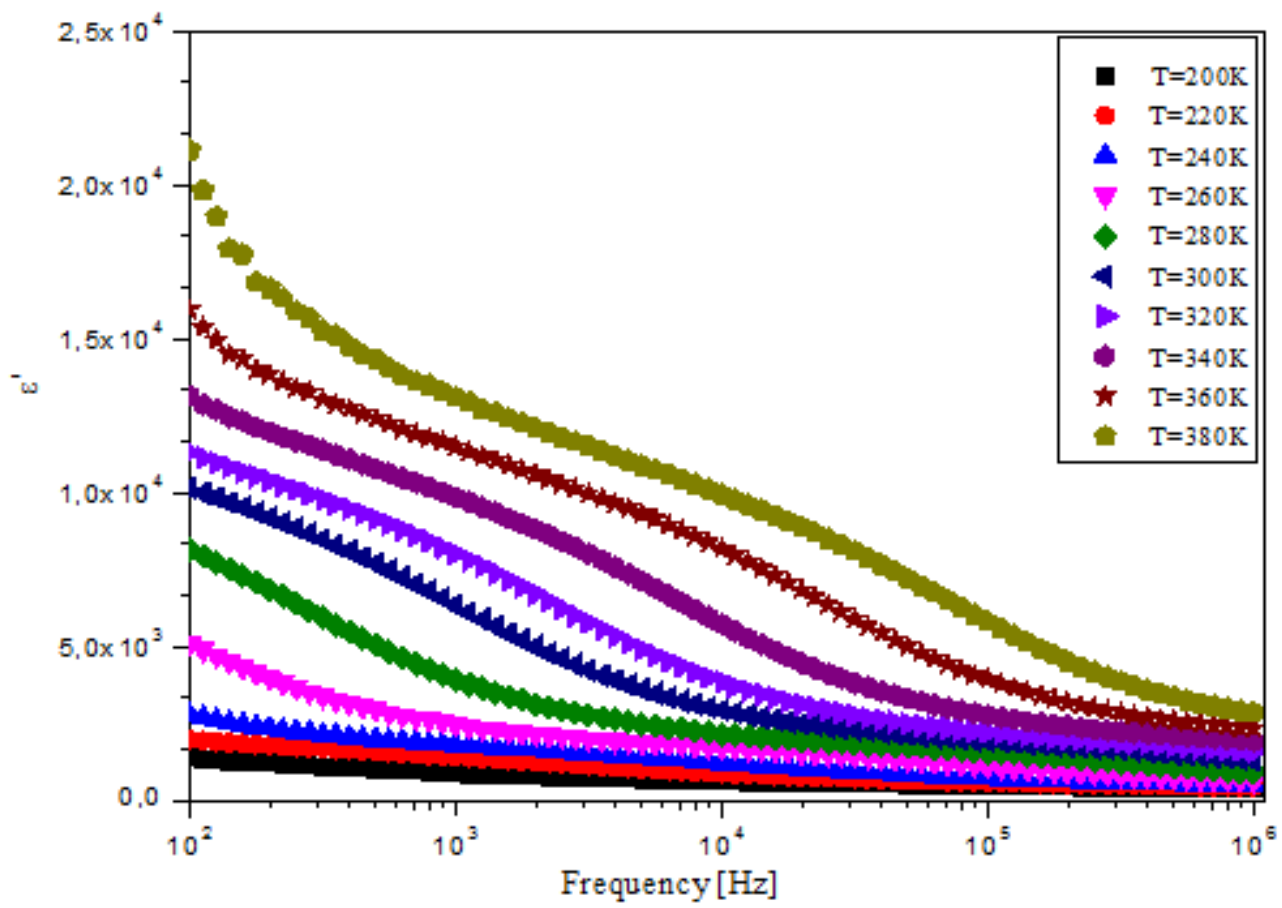




\section{Figure 8}

Frequency and temperature dependencies of the dielectric constant of LaPbFeTiO6.

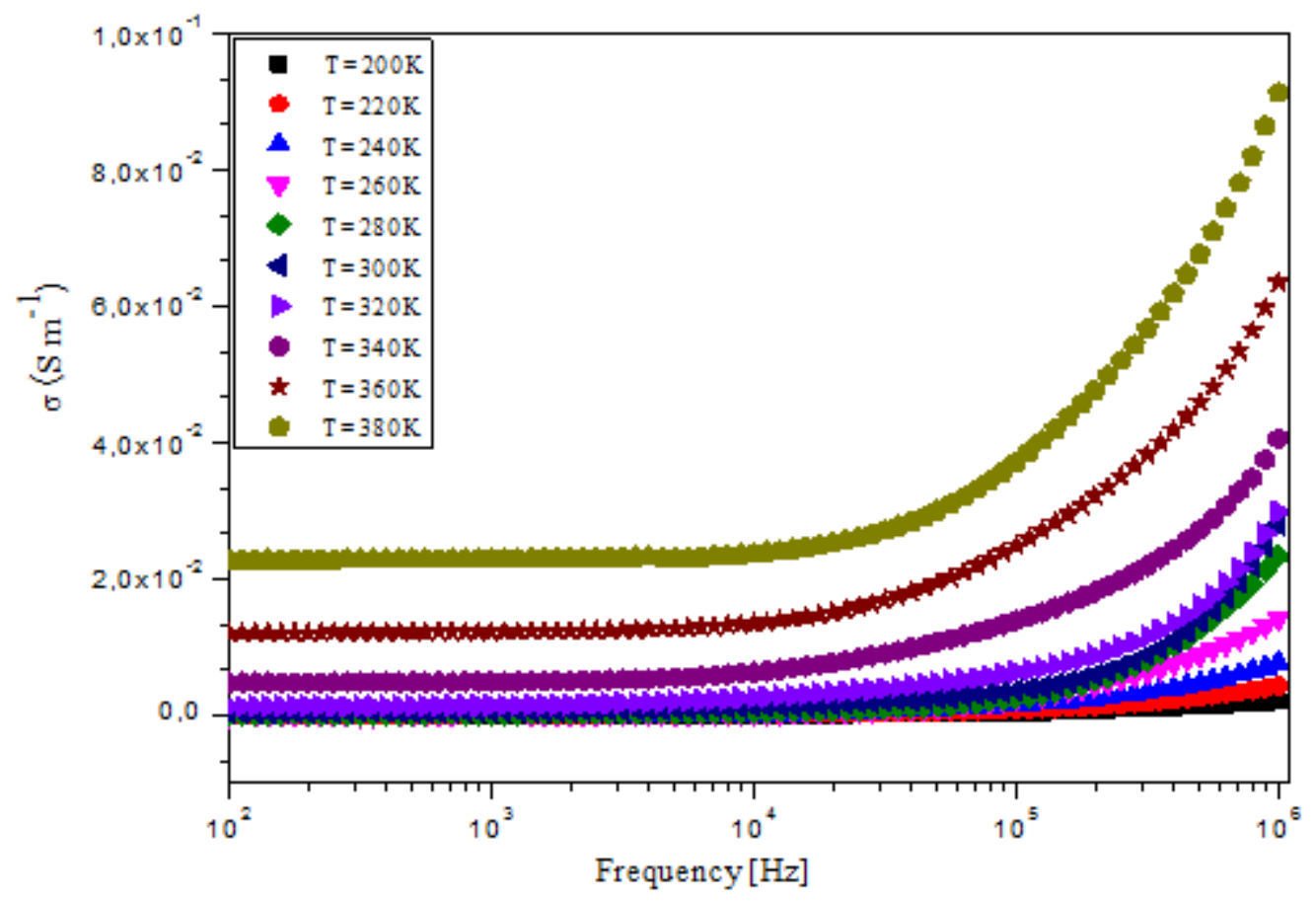

Fig.9

\section{Figure 9}

Frequency and temperature dependencies of the electrical conductivity of the LaPbFeTiO6. 


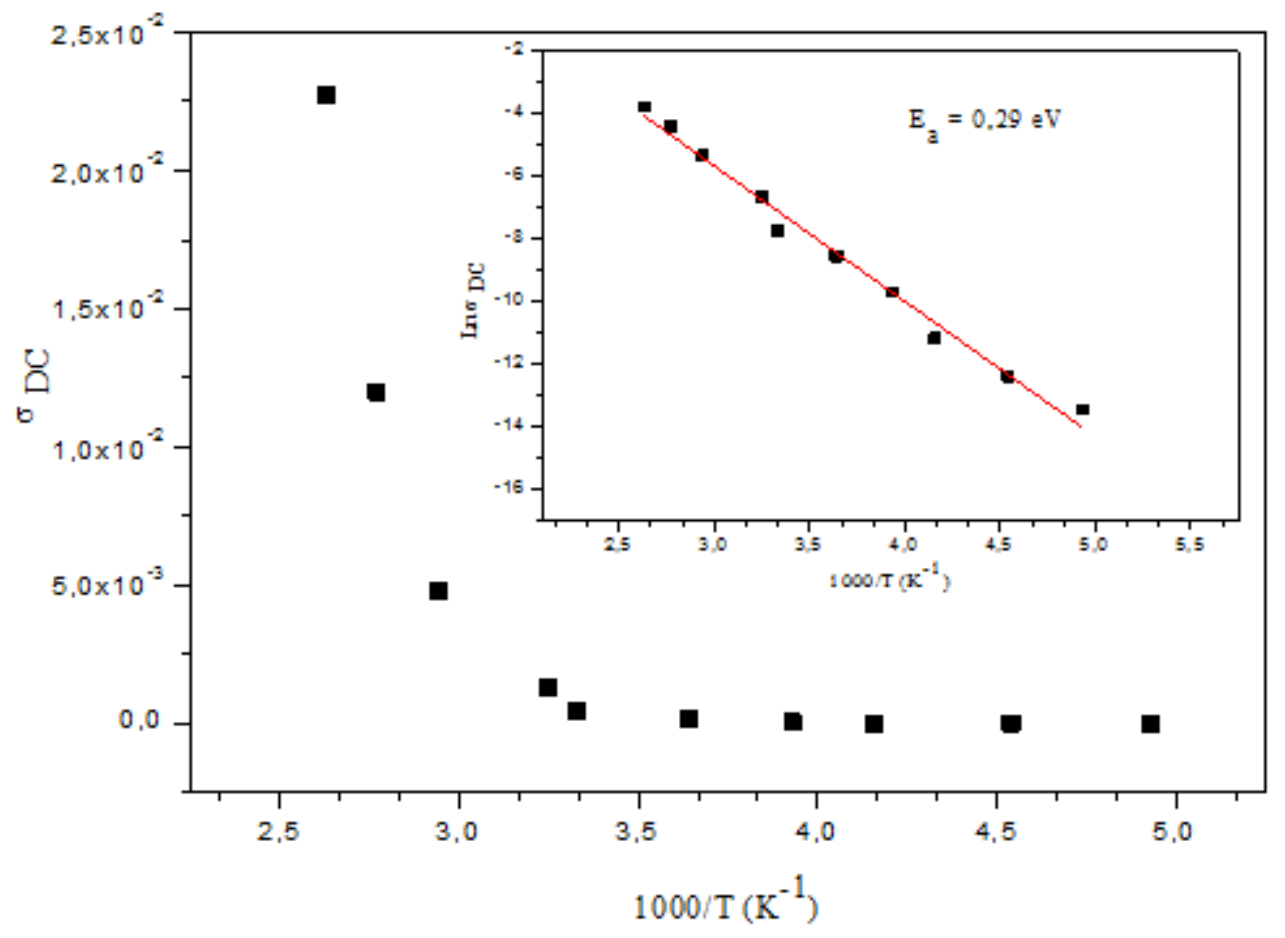

Figure 10

Temperature dependence of the DC conductivity of the LaPbFeTiO6 ceramic and the linear In ( $\left.\sigma \_D C\right)$ as a function of 1000/T, which satisfy the Arrhenius law. 


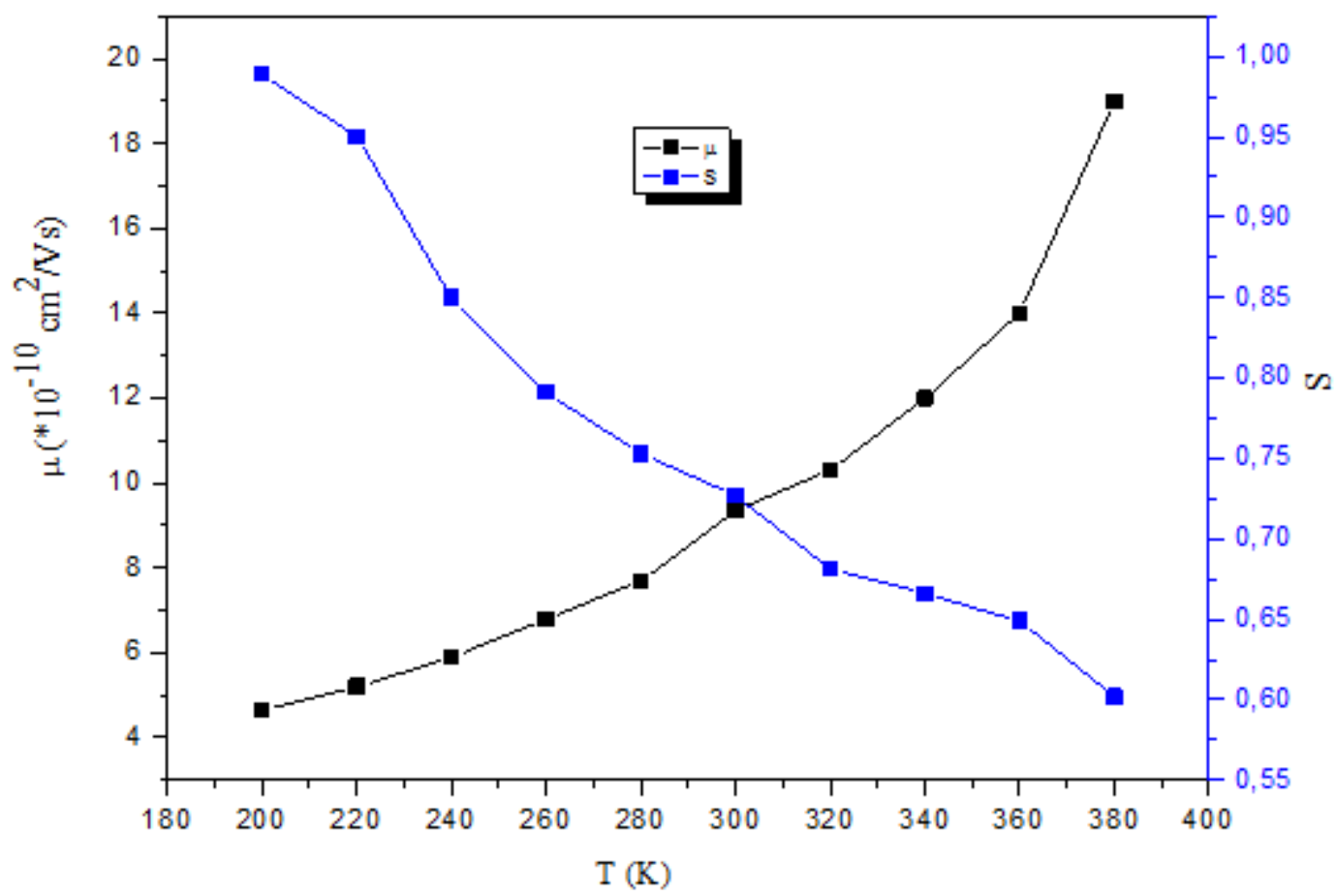

Fig.11

Figure 11

Temperature dependences of the charge carriers mobilities and the exponent s of the LaPbFeTiO6.

\section{Supplementary Files}

This is a list of supplementary files associated with this preprint. Click to download.

- Table.pdf 